\title{
The Effects of Concentration, Temperature and Solvent Composition on Partial Molar Volumes of Cetyltrimethylammonium Bromide in Methanol- Water mixed Solvent Media
}

\author{
Sujit Kumar Shah, Ajaya Bhattarai, Ghanshyam Shrivastav and Sujeet \\ Kumar Chatterjee*
}

Dept. Chemistry, Mahendra Morang A. M. Campus, Tribhuvan University, Biratnagar, Nepal. e-mail: sujeetkumarchatterjee@yahoo.com

\begin{abstract}
Precise measurements on the density of cetyltrimethylammonium bromide solutions in pure water and in methanol(1) + water (2) mixed solvent media containing 0.10, 0.20 and 0.30 volume fractions of methanol at $308.15,318.15$ and $323.15 \mathrm{~K}$ are carried out to calculate the partial molar volumes. The concentrations are varied from $0.3-1.0 \times 10^{-1}$ mol. $l^{-1}$. The results showed almost constant on the partial molar volumes with increasing surfactant concentration. Also, the partial molar volumes are found to increase with increasing temperature over the entire concentration range investigated in a given mixed solvent medium and these values are found to decrease with increasing methanol content in the solvent composition.
\end{abstract}

Keywords: Mixed solvent, cetyltrimethylammonium bromide, surfactant, partial molar volume.

\section{Introduction}

Surfactant is a species that accumulates at the interface of two phases or substances and modifies the properties of the surface. Many surfactants play an important role in the field of science and technology due to their unique properties. The cetyltrimethylammonium bromide (CTAB) is bio-degradable, non-toxic and is widely available material at low costs.

It is widely used in the food processing, to stabilize emulsions and suspensions, and to improve the texture of the food products. The objective of the present investigation would be to study the density of cetyltrimethylammonium bromide (CTAB) in solution phase. This study is expected to be very important to make fruitful use of this class of surfactants, i.e. either to develop new applications or to extend already existing applications. In particular, the behavior of the cetyltrimethylammonium bromide (CTAB) will be investigated in

${ }^{*}$ Corresponding author 
different methanol-water mixtures under salt free condition. In mixed solvent systems, however, the dielectric constants of the media can be varied at will simply by changing the solvent-composition. The organic solvent will be chosen in such a way that the polarities of the mixed solvent media do not change with composition

The micelle formation of surfactants in solutions has been known to be affected by the temperature, the pressure and various kinds of additives. Studies of the effect of organic additives on the critical micelle concentration (CMC) have led to an understanding of the part played by the solvent in the micelle formation. ${ }^{1-6}$ The thermodynamic quantity known as the partial molar volume has proved to be very useful tool in elucidating the interactions occurring in solutions. Studies of partial molar volume of surfactants have been used to examine the behavior of surfactant solutions. ${ }^{7-12}$ With regard to the partial molar volume in a mixed solvent, Lee and Hyne ${ }^{13}$ have been determined the partial molar volumes of tetraalkylammonium chlorides in ethanol-water mixtures in order to examine ion-solvent interactions in aqueous solvent mixtures. They have also discussed the structural changes accompanying the addition of ethanol to water.

In this paper, the results are reported for density measurements on cetyl trimethylammonium bromide, a cationic surfactant, in methanol-water mixed solvent media with varying relative permittivity at different temperatures. A thorough knowledge of the solution behavior of surfactant deserves information on a variety of physical and chemical parameters. The partial molar volume is one of the important thermodynamic properties which may be helpful in the identification of solvent - solute as well as solute - solute interactions. ${ }^{14}$ Moreover, this partial molar volume is necessary for the application of characterization methods and physicochemical techniques including analytical and preparative ultracentrifugation, as well as small - angle X-ray and neutron scattering. Recently empirical procedures have been developed to calculate and predict the partial molar volume for non-ionic and ionic organic compounds in aqueous solutions. ${ }^{15,16}$ However, the evaluation of these procedures for surfactant is limited by the lack of availability of reliable experimental data of a broad variety of chemical structures and macromolecular characteristics. The effects of concentration, temperature and solvent composition on the partial molar volume have been studied for the surfactant cetyltrimethylammonium bromide. Detailed definitions and explanation of the partial molar volume have been provided in several papers. ${ }^{17-19}$ Hence, only basic relation pertaining to the system studied herewith will be dealt. The partial molar volume, $\bar{V}$, is defined by the following equation;

$$
\bar{V}=(\partial V / \partial n)_{T, p}
$$

where, $\partial V$ represent change in total volume and $n$ as the number of moles. The partial molar volume is often provided in units of partial molar volume $\mathrm{cm}^{3} / \mathrm{mol}$. If there is concentration dependence, the partial molar volumes have to be extrapolated to concentration zero using the following equation which calculate the apparent molar volume at the finite concentrations (c). ${ }^{17}$

$$
\bar{V}=\frac{M}{\rho_{0}}-\frac{10^{3}}{c}\left(\frac{\rho}{\rho_{0}}-1\right)
$$


where, $\mathrm{M}$ is the molecular weight of the Cetlytrimethylammonium bromide, $\rho_{0}$ is the density of the solvent, $\rho$ is the density of the solution and $\mathrm{c}$ is having the unit as equivalent concentration in $\mathrm{mol} / \mathrm{L}$.

The aim of the present work is to analyze the influence of concentration, medium and temperature on the surfactant solution in mixed solvent media by partial molar volumes. The solution densities are systematically measured for cetlytrimethylammonium bromide and the partial molar volume of the surfactant is computed at the temperatures $308.15,318.15$ and $323.15 \mathrm{~K}$.

\section{Experimental Methods}

Methanol (E. Merck, India, 99\% pure) was distilled with phosphorous pentoxide and then redistilled over calcium hydride. The purified solvent had a density of $0.7772{\mathrm{~g} . \mathrm{cm}^{-3}}^{-}$and a co-efficient of viscosity of $0.4742 \mathrm{mPa} . \mathrm{s}$ at $308.15 \mathrm{~K}$; these values are in good agreement with the literature values. ${ }^{20}$ Triply distilled water with a specific conductance less than $10^{-6} \mathrm{~S}_{\mathrm{cm}} \mathrm{cm}^{-1}$ at $308.15 \mathrm{~K}$ was used for the preparation of the mixed solvents. The physical properties of methanol-water mixed solvents used in this study at $308.15,318.15$, and $323.15 \mathrm{~K}$ are reported in Table 1 have been taken from the published papers. ${ }^{26}$ The relative permittivity of methanol-water mixtures at the experimental temperatures were obtained by regressing the relative permittivity data as function of solvent composition from the literature ${ }^{22}$ and are included in Table 1.

Table 1: Properties of methanol(1) + water (2) mixed solvent media containing 0.10, 0.20 and 0.30 volume fractions of methanol at $308.15,318.15$, and $323.15 \mathrm{~K}^{26}$

\begin{tabular}{|lrrr|}
\hline $\mathrm{T} / K$ & $\rho_{0} / \mathrm{g} . \mathrm{cm}^{-3}$ & $\begin{array}{r}\eta_{0} / \mathrm{mPa} . \mathrm{s} \\
0.10 \text { methanol }\end{array}$ & $\mathrm{D}$ \\
308.15 & 0.9797 & 0.8665 & 71.57 \\
318.15 & 0.9760 & 0.7017 & 68.18 \\
323.15 & 0.9741 & 0.6375 & 66.45 \\
& & 0.20 methanol & \\
308.15 & 0.9663 & 1.0217 & 68.14 \\
318.15 & 0.9616 & 0.8075 & 64.80 \\
323.15 & 0.9588 & 0.7300 & 63.15 \\
& & 0.30 methanol & \\
308.15 & 0.9516 & 1.1418 & 64.25 \\
318.15 & 0.9463 & 0.8957 & 60.99 \\
323.15 & 0.9433 & 0.8052 & 59.41 \\
\hline
\end{tabular}

Cetlytrimethylammonium bromide (CTAB) employed in these investigations was purchased from Aldrich Chemical Company, Inc. To measure density the pycnometeric method was used. The stock solutions were freshly prepared for each concentration series to 
avoid problems of aging and microorganism contamination, which was found to occur with diluted surfactant solutions. ${ }^{23}$

The densities of solutions were determined by the use of Ostwald-Sprengel type pycnometer of about $10 \mathrm{~cm}^{3}$ capacity. The sample solution was transfused into the pycnometer by using a medical syringe. The pycnometer was then tightly fixed in a thermostat at the experimental temperatures within $\pm 0.005 \mathrm{~K}$. After thermal equilibrium was attainded, the mass of the pycnometer was measured with electronic balance and the density was calculated. Density measurements are believed to be precise within \pm 0.00005 , which is satisfactory for our purpose. In order to avoid moisture pickup, all solutions were prepared in a dehumidified room with utmost care. In all cases, the experiments were performed at least in three replicates.

We determined the partial molar volume by using equation (2), for example the molecular weight of Cetyltrimethylammonium bromide(M) is 364.43 , the density of the solvent (pure water) $\rho_{0}=0.99406$ at $308.15 \mathrm{~K}$ which was obtained from the literature ${ }^{24}$, the density of the solution $(\rho)=0.99436$ at $308.15 \mathrm{~K}$ which was determined from the measurement of the weight of the solution of Cetyltrimethylammonium bromide in methanol water mixed solvent media and the concentration of the solution(c $)=0.09953$ $\mathrm{mol} / \mathrm{L}$. By putting all these values in the given equation (2) we obtained $363.57 \mathrm{~cm}^{3} / \mathrm{mol}$, which is the partial molar volume.

\section{Results and Discussion}

The partial molar volumes for the Cetyltrimethylammounium bromide in pure water and in three different methanol-water mixtures (containing 0.10, 0.20, and 0.30 mole fraction of methanol) at 308.15, 318.15 and 323.15 K are depicted in Figs 1, 2 and 3, respectively, which show the variation of partial molar volumes of the investigated solutions as a function of the surfactant concentration. From these results, it is evident that the partial molar volumes exhibit almost constant with increasing concentration within the concentration range investigated here. These results are in agreement with equation (2) and it was observed in our investigation that in the concentration range above critical micelle concentration (CMC), the partial molar volumes of Cetyltrimethylammonium bromide in methanol water mixed solvent media found to be almost constant. The similar behavior was also reported in the methanol water mixed solvent media for polystyrenesulphonate. ${ }^{26}$ Furthermore, our partial molar volume data for pure water of cetyltrimethylammounium bromide match with the previously published paper. ${ }^{24}$ Obviously, the concentration independence of partial molar volumes follows one and the same pattern at all the temperatures and solvent composition investigated.

The effects of temperature and relative permittivity on the partial molar volume values are directly evident from figures 1,2 and 3. At each temperature, the partial molar volume values are found to decrease with decreasing relative permittivity in going from pure water up to 0.30 mole fraction of methanol over the entire concentration range investigated. An increase in temperature, on the other hand, is found to increase the partial molar volume in a 
given solvent medium as manifested in these figures. This is because with increasing temperature the contribution from the surfactant-solvent binding is weakened and the partial molar volume of the surfactant increases significantly with temperature. ${ }^{25}$

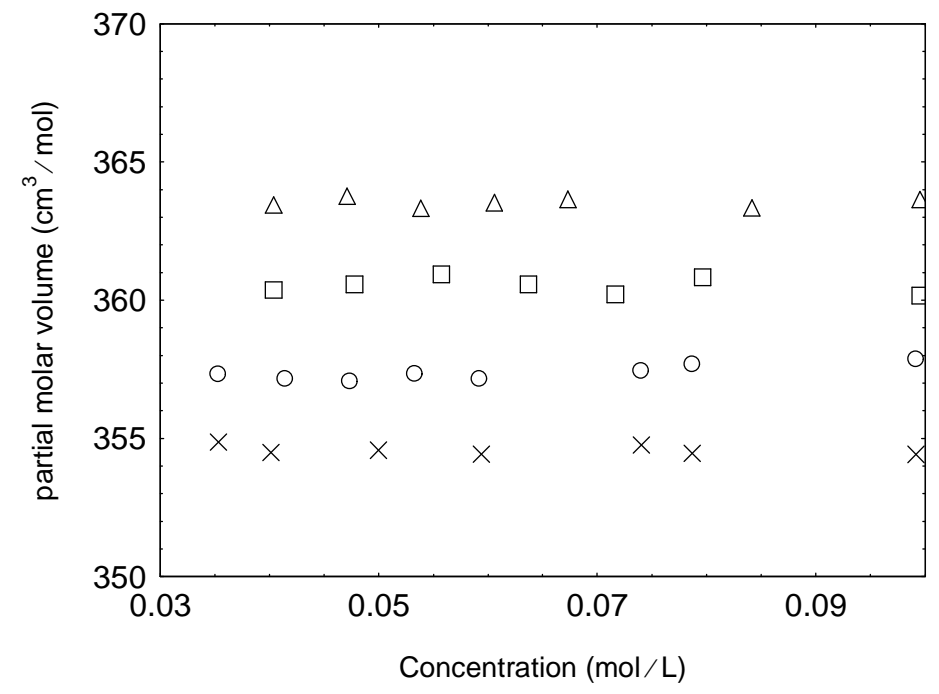

Figure 1: Concentration independence of partial molar volume for cetyltrimethylammounium bromide at $308.15 \mathrm{~K}$, in pure water (triangles) and different methanol-water mixtures (squares, 0.10 methanol; circles, 0.20 methanol; crosses, 0.30 methanol).

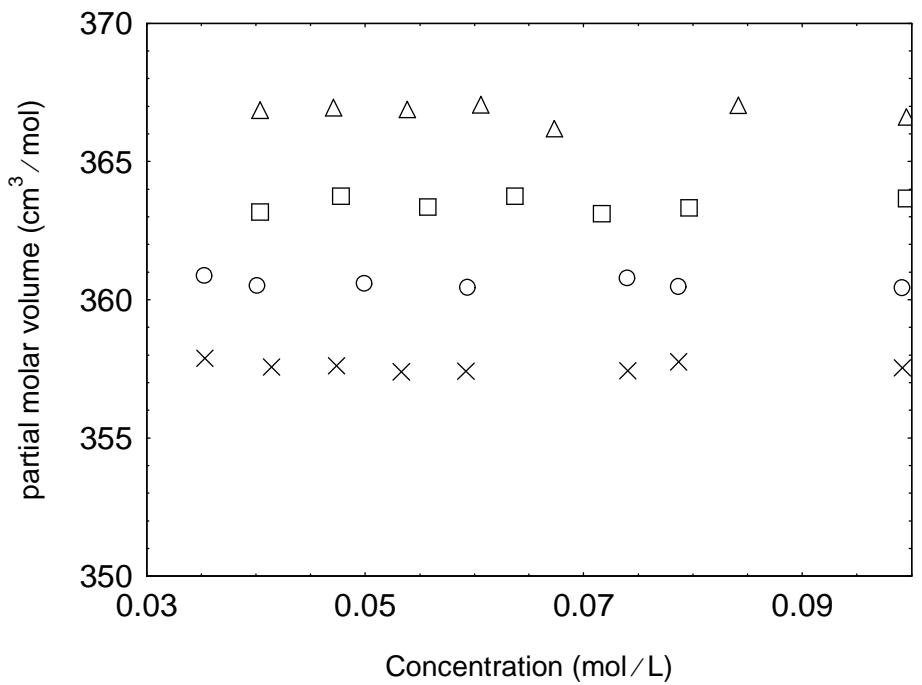

Figure 2: Concentration independence of partial molar volume for cetyltrimethylammounium bromide at $318.15 \mathrm{~K}$, in pure water (triangles) and different methanol-water mixtures (squares, 0.10 methanol; circles, 0.20 methanol; crosses, 0.30 methanol). 


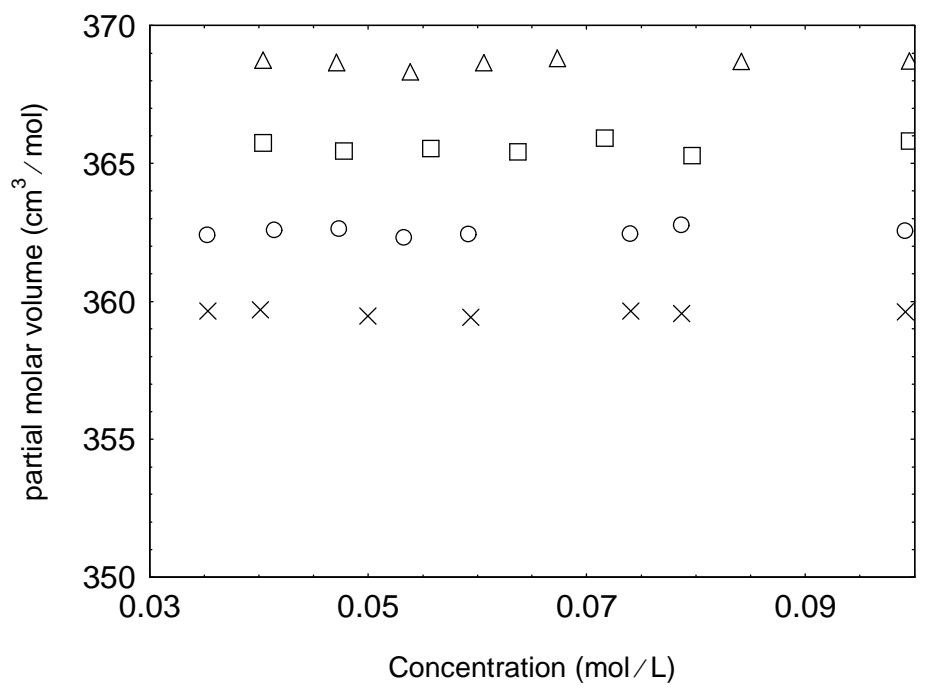

Figure 3: Concentration independence of partial molar volume for cetyltrimethylammounium bromide at $323.15 \mathrm{~K}$, in pure water (triangles) and different methanol-water mixtures (squares, 0.10 methanol; circles, 0.20 methanol; crosses, 0.30 methanol).

The relative permittivity of the medium decreased at a given temperature with increasing in the methanol content and this also been established from our earlier conductivity study. ${ }^{21}$ Lower relative permittivity promotes greater counterion-binding, and hence a lower amount of uncondensed counter ions in going from 0.10 and 0.30 mole fraction of methanol in the mixed solvent media over the entire range of temperatures investigated.

From our study, the partial molar volumes of Cetyltrimethylammonium bromide in methanol water mixed solvent media was found to be almost constant within the concentration range $\left(0.3-1.0 \times 10^{-1}\right.$ mol. $\left.\mathrm{L}^{-1}\right)$ above critical micelle concentration $(\mathrm{CMC})$. The nature of the graphs as shown in Figs 1,2 and 3 are almost same as in the case of Sodium Polystyrenesulphonate in methanol water mixed solvent media in the concentration range $\left(1.0-3.8 \times 10^{-2} \mathrm{monomol} / \mathrm{L}\right)^{26}$, although Cetyltrimethylammonium bromide is a cationic surfactant and Sodium Polystyrenesulphonate is a polyelectrolyte, which is an interesting aspect of our study. The only difference we observed in both cases is the values of partial molar volumes, due to the difference in their molecular weights and densities.

\section{Conclusions}

Results for the partial molar volumes of salt-free solution of a surfactant cetyltrimethylammonium bromide in methanol-water mixed solvent media are presented as a function of surfactant concentration and temperature. The partial molar volumes are found to increase with increasing temperature over the entire concentration range 
investigated in a given mixed solvent medium. On the other hand, partial molar volume values at a particular temperature are found almost same even at the different concentration of the medium and these values are found to decrease as the relative permittivity of the medium decreases.

\section{Acknowledgements}

This work was supported by a Research Grant for the fiscal year 2008/2009 of Nepal Academy of Science and Technology (NAST), Kathmandu, Nepal.

\section{References}

1. M. F. Emerson, A. Holtzer, J. Phys. Chem., 1967, 71, 3320.

2. G. D. Parfitt, J. A. Wood, Kolloid-Z. Z. Polym., 1969, 55, 229.

3. K. Shirahama and T. Kashieabara, J. Colloid Interface Sci., 1971, 36, 65.

4. K. Deguchi, T. Mizuno, K. Meguro, J. Colloid Interface Sci., 1974, 48, 474.

5. J. W. Larsen, L. B. Tepley, J. Colloid Interface Sci., 1974, 49, 113.

6. N. Nishikido, Y. Moroi, H. Uehara, R. Matuura, Bull.Chem.Soc. Jpn., 1974, 47, 2634.

7. K. Shinoda,T. Soda, J. Phys. Chem., 1963, 67, 2072.

8. J.M. Corkill, J.F. Goodman, T. Walker, Trans. Faraday Soc., 1967, 63, 768.

9. J. E. Desnoyers, M. Arel, Can. J. Chem., 1967, 45, 359.

10. G. M. Brown, P. Dubreuil, F. M. Ichhaporia, J. E. Desnoyers, Can. J. Chem., 1970, 48, 2525.

11. G. M. Musbally, G. Person, J. E. Desnoyers, , J. Colloid Interface Sci., 1974, 48, 494.

12. P. A. Leduc, J. L Fortier, J. E. Desnoyers, J. Phys. Chem., 1974, 78, 1217.

13. I. Lee, J.B. Hyne, Can. J. Chem., 1968, 46, 2333.

14. N. Ise, T. Okubo, J. Am. Chem. Soc., 1968, 90, 4527.

15. H. Durchschlag, P. Zipper, Prog. Colloid Polym. Sci., 1994, 94, 20.

16. P. Gianni, L. Lepori, J. Solution Chem., 1996, $25,1$.

17. H. Eisenberg, in Biological Macromolecules and polyelectrolytes in Solution; Clarendron, Oxford. 1976.

18. H. Durchschlag, in Thermodynamic Data for Biochemistry and Biotechnology (ed. Hinz, H.-J.) Springer, Berlin. 1986, p. 45.

19. R. L. Rowell, Colloids Surf., 1986, 21, 279.

20. G. Moumouzias, D. K. Panopoulos, G. Ritzoulis, J. Chem. Eng. Data, 1991, 36, 20.

21. A. Bhattarai, P. Nandi, B. Das, J. Polym. Research, 2006, 13, 475.

22. P. S. Albright, L. J. Gasting J. Am. Chem. Soc., 1946, 68, 1061.

23. A. Domard, M. Rinaudo, Int. J. Biol. Macromol, 1983, 5, 49.

24. R. De Lisi, S. Milioto, R. E. Verrall, J.Solution Chem., 1990, 19,7.

25. M. J. Iqbal, M. Siddiquah, J.Braz. Chem. Soc., 2006, 17,851.

26. A. Bhattarai, B. Das, J. Nepal Chem. Soc., 2008, 23, 82. 